\title{
Adenosquamous carcinoma of the hepatic flexure of colon: a case report: a case report
}

\author{
Bo Huang ${ }^{1}$, Hongmei $\mathrm{Li}^{1,2}$, Ping $\mathrm{Li}^{3}$ \\ ${ }^{1}$ The People's Hospital of Shapingba District in Chongqing, Chongqing, China; ${ }^{2}$ Oncology Department, Southwest Hospital of Third Military \\ Medical University (Army Medical University), Chongqing, China; ${ }^{3}$ Oncology Department, West China Hospital of Medicine, Sichuan University, \\ Chengdu, China \\ Correspondence to: Hongmei Li. Oncology Department, Southwest Hospital of Third Military Medical University (Army Medical University), \\ Chongqing 400038, China. Email: lhm211985@126.com; Ping Li. Oncology Department, West China Hospital of Medicine, Sichuan University, \\ Chengdu, 610041, China. Email: leepingmd68@vip.163.com.
}

\begin{abstract}
Adenosquamous carcinoma (ASC) is defined as a very rare subset of colorectal cancer containing both adenocarcinoma (AC) and squamous cell carcinoma (SCC) components. ASC is more aggressive for resulting in a poorer prognosis than $\mathrm{AC}$ alone. To date, only few cases were recorded previously. Here, we reported that a 46-year-old woman was caught by abdominal pain and hematochezia for more than 20 days and she visited the emergency room with increasing abdominal pain and vomiting for nearly one day. Physical examination indicated direct tenderness in the navel and lower abdomen distension. Laboratory examinations showed a decreased hemoglobin, increased white blood cell (WBC) count, an elevation of the serum levels of CA19-9 and carcinoembryonic antigen (CEA). Then endoscopic examination revealed a hemicircumferential mass in the ascending colon, which contained a friable and ulcerated lesion that bled easily when touched. Results of biopsy suggested a high-grade intraepithelial neoplasia. Abdominal computed tomography (CT) scanning showed some signs of neoplastic lesion that the wall of the hepatic curvature of the transverse colon is irregularly thicker, the serous surface of the diseased intestinal canal is slightly thicker, and multiple peripheral lymph nodes are enlarged. Furthermore, the multidisciplinary team (MDT) meeting thought laparoscopic right colectomy accompanied with lymphadenectomy was suitable because the clinical stage was at least stage IIIB. The post-operative pathological examination demonstrated that the tumor was composed of AC (positive for CDX-2 and CK20) and SCC (positive for CK5/6 and P63) components. Accordingly, this case was diagnosed as ASC of Stage IIIC (pT4N2M0) and the adjuvant chemotherapy FOLFOX was recommended, while unfortunately the patient refused it for personal reasons. In conclusion, radical resection remains the major treatment for colon ASC, CT scan and endoscopic examination and biopsy are useful for this pre-operative diagnosis. This case promotes the understanding of colon ASC and provides a basis for its clinical diagnosis and treatment.
\end{abstract}

Keywords: Colorectal cancer; adenosquamous carcinoma (ASC); hepatic flexure of colon; case report

Submitted Nov 15, 2020. Accepted for publication Mar 24, 2021.

doi: $10.21037 /$ tcr-20-3264

View this article at: http://dx.doi.org/10.21037/tcr-20-3264

\section{Introduction}

Colorectal cancer is the third most common cancer globally. Primary adenosquamous carcinoma (ASC) of the colon is extremely rare entities (1), of which the incidence is around $0.06 \%$ of all colorectal cancers (2). It was described as a cancer containing both adenocarcinoma (AC) and squamous cell carcinoma (SCC) component (3). Clinical manifestations are similar to colorectal ACs, and the diagnosis is based primarily on histologic examination (4). Surgical resection remains the primary therapy for colorectal ASC, and the prognosis of adenosquamous 

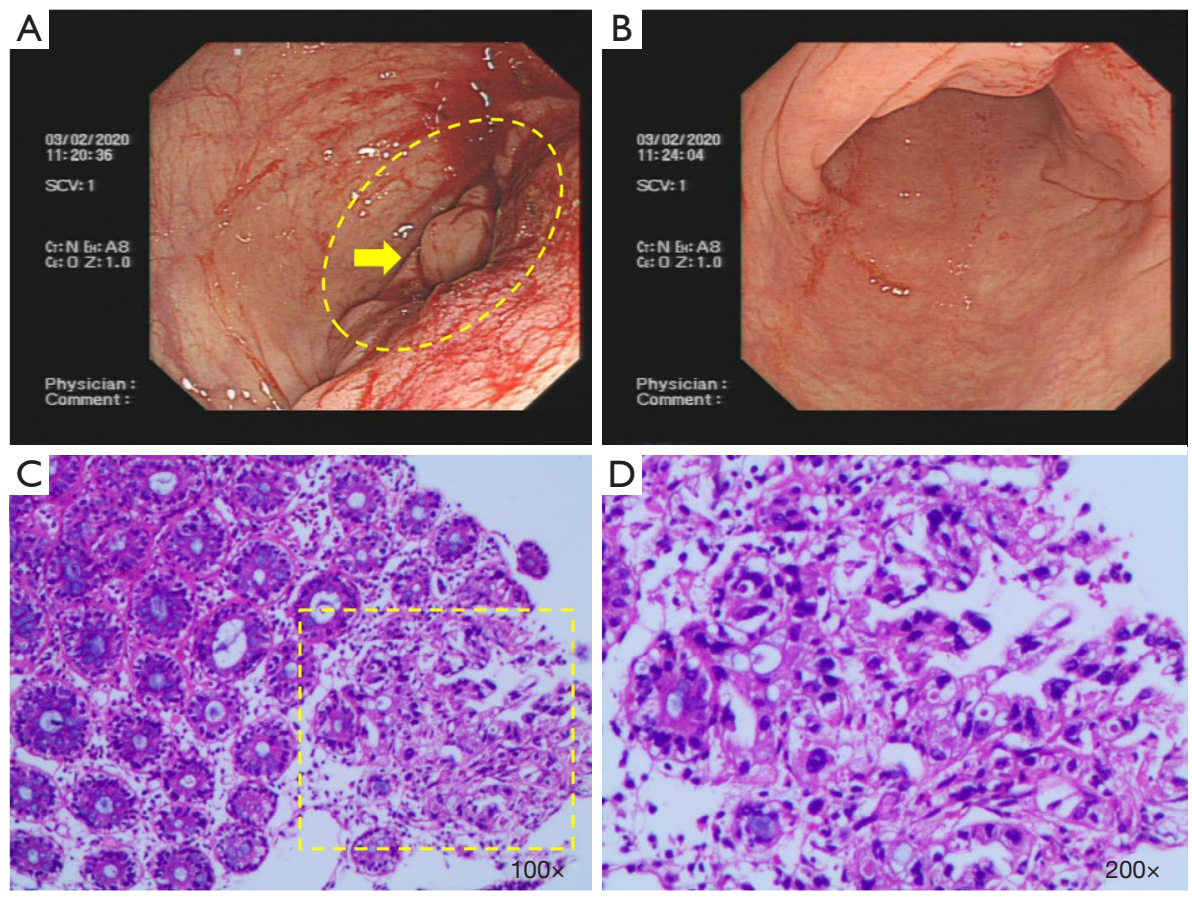

Figure 1 Colonoscopic examination of the colon. (A) Colonoscopy revealed the hemicircumferential ascending colon tumor. The residual colorectal mucosa is characterized by hyperemia and edema. (B) Normal colonic mucosa without erosion and ulceration. (C,D) Biopsy and hematoxylin-eosin staining were performed. The heterosexual glands were found on the surface of a mucous membrane, suggesting highgrade intraepithelial neoplasia. (D) (200x) shows the part of the yellow dotted frame in (C) (100x).

colon cancer is poorer than $\mathrm{AC}$ alone (5). Here, we presented a 46-year-old woman diagnosed as colon ASC through computed tomography (CT) scan and endoscopic examination with abdominal pain and hematochezia for more than 20 days, which was consistently confirmed by pathological examinations of surgical specimen. We present the following article in accordance with the CARE reporting checklist (available at http://dx.doi.org/10.21037/ tcr-20-3264).

\section{Case presentation}

A 46-year-old female experienced a history of abdominal pains and hematochezia for more than twenty days, and visited the emergency room with increasing abdominal pain and vomiting for nearly one day. She was hospitalized for large bowel obstruction syndrome. Her past medical and family history was noncontributory. Upon physical examination, direct tenderness in the navel and the lower abdomen is positive, accompanied by abdominal distension. Laboratory examinations showed a hemoglobin $10.6 \mathrm{~g} / \mathrm{dL}$, white blood cell (WBC) $14.21 \times 10^{3} / \mathrm{mm}^{3}$,
Neutrophil (Neu) $12.75 \times 10^{3} / \mathrm{mm}^{3}$. The serum levels of epithelial tumor markers, such as carbohydrate antigen 19-9 (CA19-9; $133.70 \mathrm{U} / \mathrm{mL}$ ) and carcinoembryonic antigen (CEA; $7.62 \mathrm{ng} / \mathrm{mL}$ ) were exceeding the normal limit, and fecal occult blood (OB) was also positive.

During the endoscopic procedure, it was available to explore the gut from the anus to the hepatic flexure of transverse colon $(70 \mathrm{~cm}$ from the anal margin), where a tight stenosis blocking the endoscopic passage was found with a hemicircumferential mass in the ascending colon. The mass contained a friable and ulcerated lesion, and it bled easily when touched. The residual colorectal mucosa is characterized by hyperemia and edema (Figure 1A,B). Biopsy was performed. Microscopically, the pathological result was chronic inflammation of the mucous membrane in superficial erosion. Heterosexual glands were found on the surface of a mucous membrane, suggesting highgrade intraepithelial neoplasia (Figure 1C,D). Abdominal computed tomography (CT) scanning showed that the wall of the hepatic curvature of the transverse colon thickened irregularly, resulting in the dilatation of right colon, appendix and terminal ileum. Moreover, the serous 

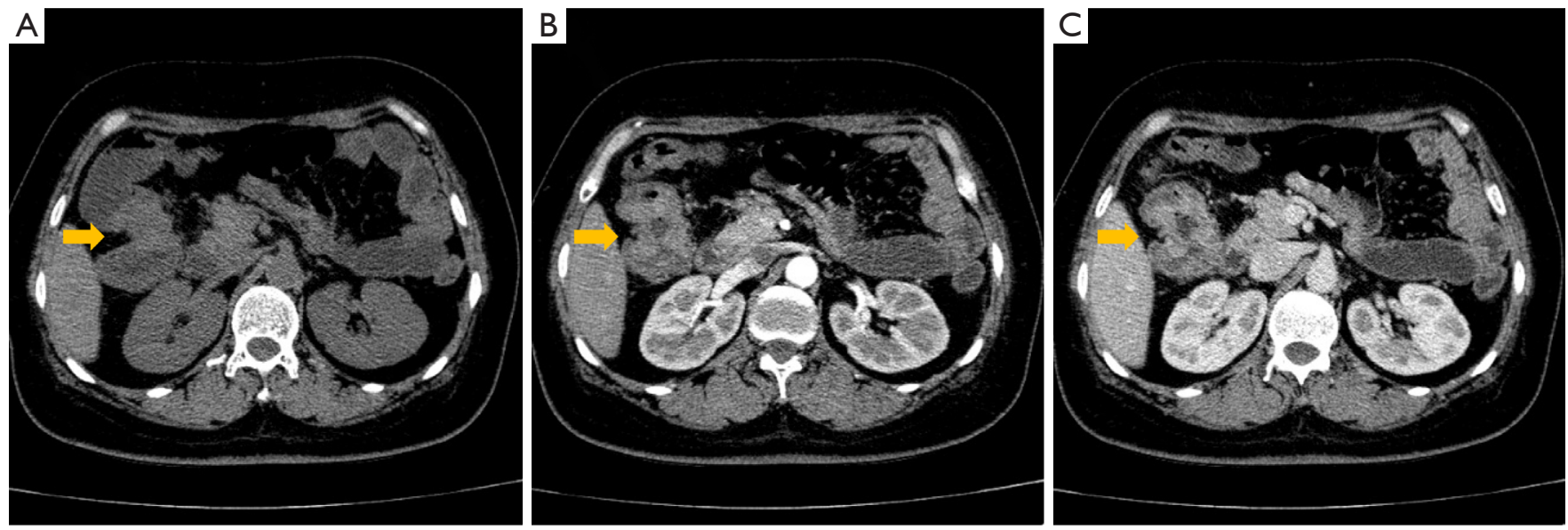

Figure 2 CT findings with contrast medium injection. Incrassation of the ascending colon wall (arrowheads) and swollen lymph nodes is shown. (A) Plain scan, (B) arterial phase, (C) venous phase.

surface of the diseased intestinal canal is slightly thicker, and multiple peripheral lymph nodes are enlarged. Thus, it could be considered as neoplastic lesions (Figure $2 A, B, C)$. In addition, no evidence of distant metastasis was found from imaging examination.

For more precise and personalized treatment, this case was discussed at the multidisciplinary team (MDT) meeting with the pathologist, radiologist, oncologist, and surgeon. According to the results of CT scan and endoscopic examination, the preoperative clinical stage was at least stage IIIB, and the laparoscopic extended radical resection of right colon cancer was recommended. Then laparoscopic right colectomy accompanied with lymphadenectomy was performed. No obvious abnormality was found in abdominal organs including liver, ovaries, as well as the peritoneum. The mass was touched in the hepatic flexure of transverse colon, with a size of about $5 \mathrm{~cm} \times 5 \mathrm{~cm}$. It was hard, light and mobile and lead to the distention of the proximal colon and ileum. It invaded the serosa layer, and the growth contracture was found. And the peripheral lymph nodes were found on the right of the middle colonic artery and the root of the superior mesenteric artery, with the size of about $1.5 \mathrm{~cm} \times 2.0 \mathrm{~cm}$ were enlarged, hard and fixed. Gross examination revealed an ulcerofungating mass with a size of $6.0 \mathrm{~cm} \times 4.0 \mathrm{~cm} \times 2.0 \mathrm{~cm}$, which occupied the $2 / 3$ of periphery of the intestine. The cancer invaded periintestinal adipose tissue and nerve. Metastatic lesion was found in mesenteric lymph nodes (5/15). No canceration was seen in the resection margin, appendix, omentum and other intestinal tubes.
The resected specimens were routinely processed and stained with hematoxylin-eosin (H\&E) staining for pathological examination. Results showed that the tumor was composed of two components, i.e., AC and SCC (Figure 3). Immunohistochemically (Figure 4), the tumor cells from glandular components were positive for caudal type homeobox 2 (CDX-2) (Figure 4A,E) and cytokine (CK) 20 (Figure 4B,F). On the contrary, the tumor cells from squamous components were positive for CK5/6 (Figure $4 C, G$ ) and P63 (Figure $4 D, H$ ). The positive ratio of $\mathrm{Ki}-67$ was $60-80 \%$, immunohistochemical evaluation of mismatch repair (MMR) genes indicated microsatellite stability (MSS). Furthermore, according to the eighth edition of the colon and rectum cancer staging system published by the American Joint Committee on Cancer, the tumor located hepatic flexure of colon was diagnosed as ASC of Stage IIIC (pT4N2M0). After the operation, the patient recovered smoothly, but refused follow-up anti-tumor therapy for personal reasons.

All procedures performed in studies involving human participants were in accordance with the ethical standards of the institutional and/or national research committee(s) and with the Helsinki Declaration (as revised in 2013). Written informed consent was obtained from the patient.

\section{Discussion}

Colorectal ASC is a really rare entity. It is reported that the mean age was 67 years old, and it preferentially occurs in men with a sex ratio of $1.34: 1$. In addition, $53 \%$ of colorectal 

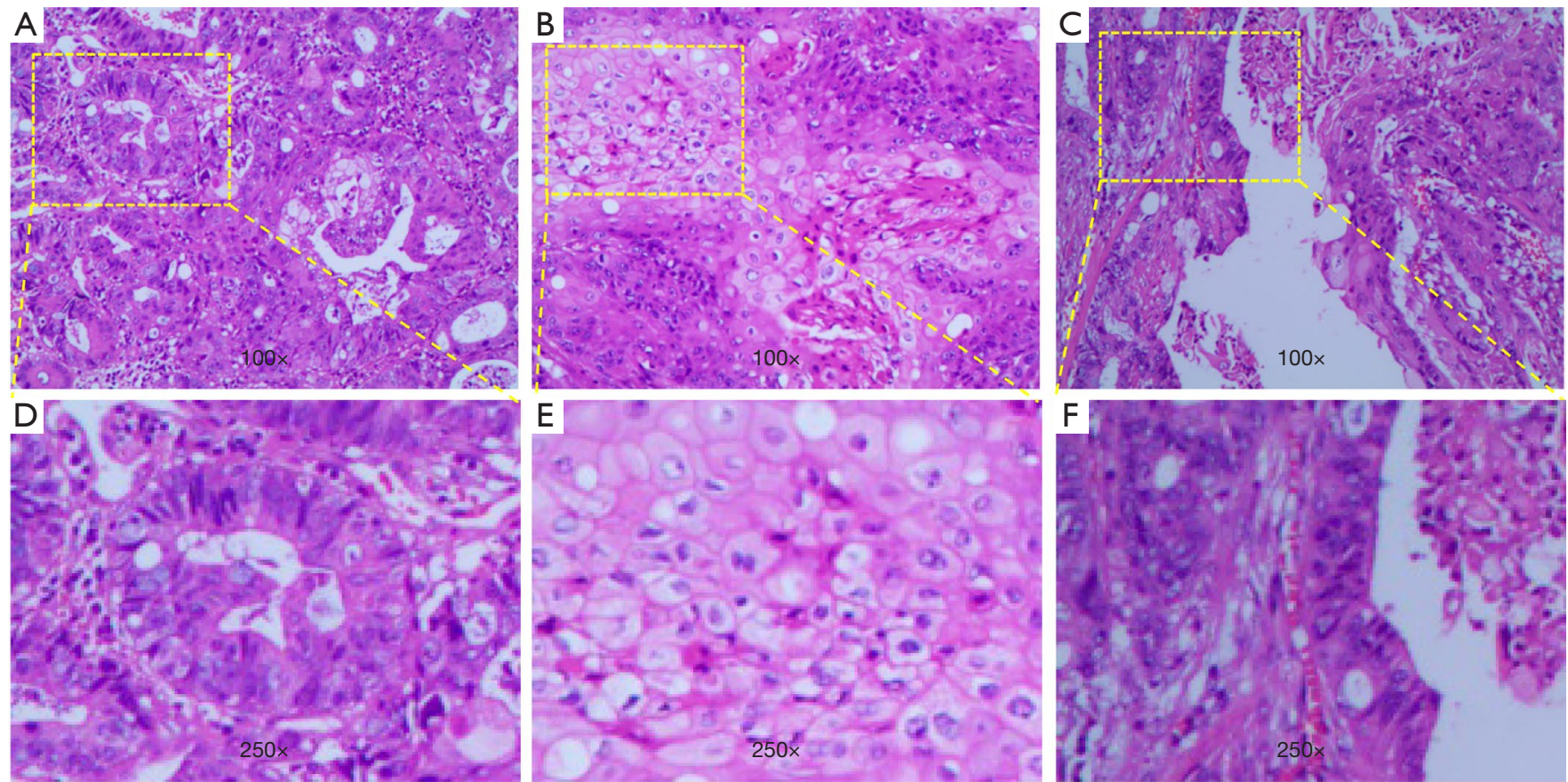

Figure 3 Microscopic findings with hematoxylin-eosin staining. (A) In a portion of the tumor, component adenocarcinoma was found ( $\times 100)$. (B) In a portion of the tumor, squamous cell carcinoma component was found $(\times 100)$. (C) In a portion of the tumor, both adenocarcinoma and squamous cell carcinoma components were found $(\times 100)$. (D-F) The corresponding part of the yellow dotted frame $(\times 250)$ in $(A-C)$, respectively.
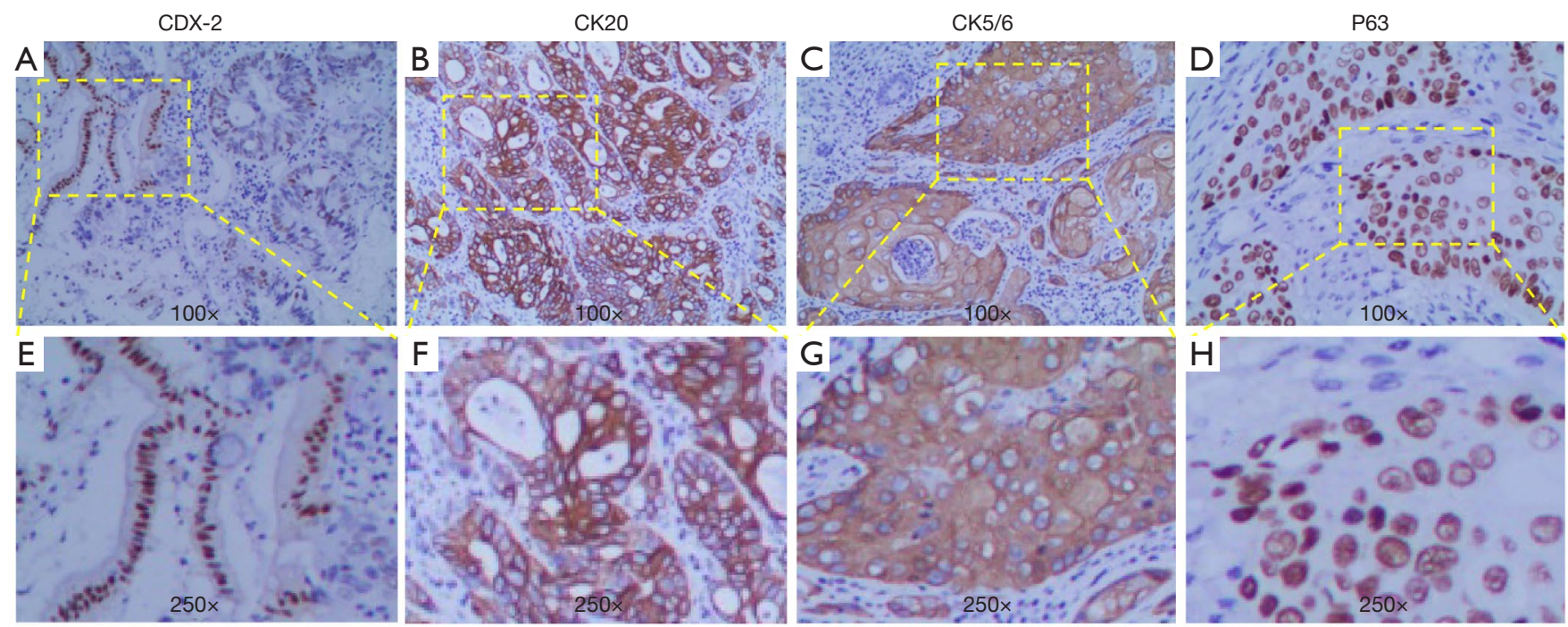

Figure 4 Findings of immunohistochemical staining. The tumor cells from glandular components were positive for CDX-2 (A,E) and CK20 $(\mathrm{B}, \mathrm{F})$. On the contrary, the tumor cells from squamous components were positive for CK5/6 $(\mathrm{C}, \mathrm{G})$ and $\mathrm{P} 63(\mathrm{D}, \mathrm{H})$. $(\mathrm{E}-\mathrm{H})(\times 250)$ showed the corresponding part of the yellow dotted frame in (A-D) $(\times 100)$, respectively. 
AC were located in the sigmoid colon, rectum, and anus (2), ASC of the colon and rectum is usually located in the right colon (6), which is consistent in this case of colon ASC originated in hepatic flexure. The prognosis of colonic ASC is worse than AC that the 5-year survival rates of ASC and AC are approximately $30 \%$ and $50-60 \%$, respectively $(7,8)$. As reported, the squamous cell component has greater metastatic potential than the glandular cell component. Thus ASC patients may suffer from more aggressive clinical symptoms (9). The most common metastatic sites of colonic ASC are liver, peritoneum and lung, respectively (1). Here, the ASC case we reported was a 46-year-old female. It located in the ascending colon, and multiple peripheral lymph nodes were invaded, but no distant metastasis was found.

The clinical signs and symptoms of ASC are very similar to those of patients with colon AC, including changes in bowel habits, abdominal pain, hematochezia, weight loss and so on. It has been demonstrated that patients with ASC may be caught by paraneoplastic syndromes such as hypercalcemia (10). However, no hypercalcemia was found in this case. As for the clinical stages of colonic ASC reported previously, $94 \%$ were T3 or more, $62 \%$ had lymph node metastasis, and $33 \%$ were stage IV (6). The case here is stage IIIC (pT4N2M0), which is consistent with the reported data. For the diagnosis of colonic ASC, a complete colonoscopy or CT colonoscopy is required. The ASC consists of two malignant components: glandular cells with different degrees of differentiation and SCC with horny pearls and intercellular bridges. Thus, histological findings based on H\&E staining are the gold standard diagnosis of primary ASC (11). It is reported that CK5/6 and P63 are specific biomarkers for SC cells, which can highlight the squamous epithelial components and favor the diagnosis of ASC or SCC (12). In our case, we stained the excised tissue with both CK5/6 and P63 antibody and identified the SC components in the ASC tissue. Generally, ASC is divided into two subgroups: the composite type (haphazard mixture of AC and SCC), and the collision type (coexistence of two adjacent but histologically distinct AC and SCC in the same organ) (13). And the case reported here is a collision one.

So far, the pathogenesis of ASC of the colon is not completely understood. The abnormal and repeated mucosal stimulus from ulcerative colitis, schistosomiasis, radiation, and human papilloma virus (HPV), can aberrantly stimulate the transdifferentiation of uncommitted cells to squamous epithelium $(14,15)$. The occurrence of colorectal ASC is also related to previous or synchronous colorectal
AC (16). Microsatellite instability (MSI) is a hypermutable phenotype responsible for the loss of DNA MMR activity $(11,17)$. MSI is detected in about $15 \%$ of all colorectal cancers. BRAF mutation in colorectal cancer is related to MSI through its association with high-level CpG island methylator phenotype (CIMP) and MLH1 promoter methylation. MSI and BRAF mutation detections are widely used for familial cancer risk assessment (18). The BRAF gene frequently mutates in colorectal cancer (19), which indicates the poor prognosis of colorectal cancer (20). In 2017, a BRAF V600E mutation was first reported on ascending colon ASC, while its sensitivity for the prognosis prediction of colonic ASC remains unknown (21).

The foremost choice for treatment of primary colonic ASC is radical surgical resection. Owing to the lack of clinical trial data, the exact effect of adjuvant radiotherapy and chemotherapy is not clear. However, chemoradiotherapy is recommended for ASC patients with lymph node metastasis (9). To date, standard chemotherapy for colonic ASC has not been established. FOLFOX (5-fluorouracil, leucovorin and oxaliplatin) adjuvant chemotherapy regimen has been used in some cases, but the exact effect of these drugs on prognosis is not clear $(11,22)$. Based on the positive detection of HPV in some cases, antiHPV infection may be an alternative adjuvant therapy (15). In the future, researches should be carried out to evaluate the effects of targeted therapy and immunotherapy in patients with stage IV colonic ASC, such as targeting the vascular endothelial growth factor (bevacizumab), epidermal growth factor receptor (cetuximab and panizumab) and immune checkpoint inhibitor (PD-1 monoclonal antibody). Furthermore, the molecular mechanisms of ASC tumorigenesis also need to be further studied.

\section{Conclusions}

In all, we reported here a rare female case of ASC in the ascending colon, with local lymph node metastasis but without distant metastasis. Preoperative diagnosis of ASC is very difficult, and should be evaluated by a MDT. This report can contribute to new knowledge for pathogenesis of colon cancer and we look forward to more in-depth researches on this rare carcinoma.

\section{Acknowledgments}

The authors would like to thank the patient.

Funding: None. 


\section{Footnote}

Reporting Checklist: The authors have completed the CARE reporting checklist. Available at http://dx.doi.org/10.21037/ tcr-20-3264

Conflicts of Interest: All authors have completed the ICMJE uniform disclosure form (available at http://dx.doi. org/10.21037/tcr-20-3264). The authors have no conflicts of interest to declare.

Ethical Statement: The authors are accountable for all aspects of the work in ensuring that questions related to the accuracy or integrity of any part of the work are appropriately investigated and resolved. All procedures performed in studies involving human participants were in accordance with the ethical standards of the institutional and/or national research committee(s) and with the Helsinki Declaration (as revised in 2013). Written informed consent was obtained from the patient.

Open Access Statement: This is an Open Access article distributed in accordance with the Creative Commons Attribution-NonCommercial-NoDerivs 4.0 International License (CC BY-NC-ND 4.0), which permits the noncommercial replication and distribution of the article with the strict proviso that no changes or edits are made and the original work is properly cited (including links to both the formal publication through the relevant DOI and the license). See: https://creativecommons.org/licenses/by-nc-nd/4.0/.

\section{References}

1. Frizelle FA, Hobday KS, Batts KP, et al. Adenosquamous and squamous carcinoma of the colon and upper rectum: a clinical and histopathologic study. Dis Colon Rectum 2001;44:341-6.

2. Cagir B, Nagy MW, Topham A, et al. Adenosquamous carcinoma of the colon, rectum, and anus: epidemiology, distribution, and survival characteristics. Dis Colon Rectum 1999;42:258-63.

3. G H. Ober heterologue cancroide. Beitr Pathol Anat 1907;41:348-412.

4. Choi JW, Park HU. Adenosquamous carcinoma of the ascending colon: a case report and review of the literature. Ann Coloproctol 2013;29:83-6.

5. Brenner H, Kloor M, Pox CP. Colorectal cancer. Lancet 2014;383:1490-502.
6. Yokoi K, Tanaka N, Furukawa K, et al. Case of adenosquamous carcinoma of the ascending colon. J Nippon Med Sch 2008;75:242-6.

7. Masoomi H, Ziogas A, Lin BS, et al. Population-based evaluation of adenosquamous carcinoma of the colon and rectum. Dis Colon Rectum 2012;55:509-14.

8. Di Prete M, Baciorri F, Franceschilli M, et al. Adenosquamous carcinoma of the right colon with diffuse signet-ring mucinous component. Clin J Gastroenterol 2020;13:555-9.

9. Shafaghi A, Askari K, Ashoobi MT, et al. Adenosquamous carcinoma of the sigmoid colon: a case report and review of literature. Int J Clin Exp Med 2013;6:390-2.

10. Chevinsky AH, Berelowitz M, Hoover HC Jr. Adenosquamous carcinoma of the colon presenting with hypercalcemia. Cancer 1987;60:1111-6.

11. Kang DB, Oh JT, Jo HJ, et al. Primary adenosquamous carcinoma of the colon. J Korean Surg Soc 2011;80 Suppl 1:S31-5.

12. Pu RT, Pang Y, Michael CW. Utility of WT-1, p63, MOC31, mesothelin, and cytokeratin (K903 and CK5/6) immunostains in differentiating adenocarcinoma, squamous cell carcinoma, and malignant mesothelioma in effusions. Diagn Cytopathol 2008;36:20-5.

13. Fukui H, Takada M, Chiba T, et al. Concurrent occurrence of gastric adenocarcinoma and duodenal neuroendocrine cell carcinoma: a composite tumour or collision tumours? Gut 2001;48:853-6.

14. Michelassi F, Montag AG, Block GE. Adenosquamous-cell carcinoma in ulcerative colitis. Report of a case. Dis Colon Rectum 1988;31:323-6.

15. Dong Y, Wang J, Ma H, et al. Primary adenosquamous carcinoma of the colon: report of five cases. Surg Today 2009;39:619-23.

16. Petrelli NJ, Valle AA, Weber TK, et al. Adenosquamous carcinoma of the colon and rectum. Dis Colon Rectum 1996;39:1265-8.

17. Duncan VE, Harada S, Stevens TM. Primary Colon Adenosquamous Carcinoma in a Patient With Lynch Syndrome: A New Histologic Subtype Associated With Microsatellite Instability? Int J Surg Pathol 2016;24:653-5.

18. Taieb J, Shi Q, Pederson L, et al. Prognosis of microsatellite instability and/or mismatch repair deficiency stage III colon cancer patients after disease recurrence following adjuvant treatment: results of an ACCENT pooled analysis of seven studies. Ann Oncol 2019;30:1466-71.

19. Davies H, Bignell GR, Cox C, et al. Mutations of the 
BRAF gene in human cancer. Nature 2002;417:949-54.

20. Lochhead P, Kuchiba A, Imamura Y, et al. Microsatellite instability and BRAF mutation testing in colorectal cancer prognostication. J Natl Cancer Inst 2013;105:1151-6.

21. Lichtenstern CR, Ngu RK, Shalapour S, et al.

Cite this article as: Huang B, Li H, Li P. Adenosquamous carcinoma of the hepatic flexure of colon: a case report. Transl Cancer Res 2021;10(5):2496-2502. doi: 10.21037/tcr-20-3264
Immunotherapy, Inflammation and Colorectal Cancer. Cells 2020;9:618.

22. Toumi O, Hamida B, Njima M, et al. Adenosquamous carcinoma of the right colon: A case report and review of the literature. Int J Surg Case Rep 2018;50:119-21. 